\title{
ETHICS
}

\section{Access and equity to cancer care in the USA: a review and assessment}

\section{A Siminoff, L Ross}

Postgrad Med J 2005;81:674-679. doi: 10.1136/pgmj.2005.032813

Cancer represents a serious threat to the health of women and men living in the USA. As the second leading cause of death, it claims about 500000 lives annually. Health disparities occur when there are differences in the incidence, prevalence, mortality, and burden of disease among specific sub-populations within a specified region. For decades, disparities have been reported among Americans from racial/ethnic minority groups and those from low income groups. African Americans, the largest racial minority group in the USA, have the highest cancer incidence and mortality rates in the USA; it is about 10\% higher in African Americans than in white people.

Inequities in insurance status among Americans adversely affect their ability to obtain the entire range of cancer care. Those who are members of ethnic minorities and the working poor are especially apt to have poorer access to care and reduced quality of cancer care services as a result.

See end of article for authors' affiliations



Correspondence to: Dr L A Siminoff, Case Western Reserve University, School of Medicine, Department of Bioethics (TA-215), 10900 Euclid Avenue, Cleveland, $\mathrm{OH}$ 44106-4976, USA las5@case.edu

Submitted 20 January 2005 Accepted

18 February 2005
C ancer represents a serious threat to the health and wellbeing of women and men living in the USA. It is the second leading cause of death, behind cardiovascular disease, claiming about 500000 lives each year. ${ }^{1}$ Cancers of the breast, lung, colon and rectum, and female reproductive organs (uterus, ovaries) account for about $65 \%$ of the new cancer cases, and $59 \%$ of the cancer deaths among American women (tables 1 and 3). ${ }^{2}$ Cancers of the prostate, lung, colon and rectum, and bladder account for about $64 \%$ of new cancer cases, and $54 \%$ of the cancer deaths among American men (tables 2 and 4). ${ }^{2}$

Not all Americans are equally at risk and many communities have disparate outcomes. Health disparities occur when there are differences in the incidence, prevalence, mortality, and burden of disease among specific population sub-groups within a specified geographical region. ${ }^{3}$ For decades, disparities in cancer incidence, prevalence, and mortality have been reported among Americans from racial/ethnic minority groups and those from low income groups.

African Americans are the largest racial minority group in the USA. They comprise slightly more than $12 \%$ of the total US population, yet they have the highest cancer incidence and mortality rates of any racial/ethnic group in the USA. ${ }^{12-7}$ The incidence rate for all cancers combined is about $10 \%$ higher in African
Americans than in white people. More alarmingly, the overall mortality rate from all cancers is about 30\% higher in African Americans than among the white population. ${ }^{2}$ The mortality rates from cancer among African American men and women are $40 \%$ and $20 \%$ higher than that of white men and women, respectively. ${ }^{7}$ Although the five year survival rate for all cancers has improved for African Americans from 27\% during 1960-1963 to 53\% during 1992-1998, this rate still lags behind that of white people, who experienced a five year survival rate increase from $39 \%$ to $64 \%$ during the same reporting period..$^{5}$ In other words, the absolute gap between white and black Americans remains unchanged.

The history of cancer disparities between Hispanics and non-Hispanic white people is not as long standing as the history of cancer disparities between the white population and African Americans. However, this disparity is troubling because Hispanics are the fastest growing ethnic minority group in the USA. By the year 2050, the US Census Bureau estimates that Hispanic will comprise $25 \%$ of the nation's population. ${ }^{8}$ Cancers that are disproportionately experienced by Hispanic Americans include cancers of the stomach, liver, and uterine cervix. The incidence of stomach cancer is at least $75 \%$ higher in Hispanics than in non-Hispanic white groups. Hispanic women have twice the cervical cancer incidence rate of non-Hispanic white women. The cervical cancer death rate for Hispanic women is about $40 \%$ higher than that of non-Hispanic white women. Hispanic women also experience twice the incidence rates of cancers of the gall bladder than non-Hispanic white women and Hispanic men. ${ }^{9}$

Although Asian/Pacific Islanders (API) and native Americans (NA) experience lower cancer rates overall compared with white people, like Hispanics, API and NA experience higher incidence and mortality rates for liver and stomach cancers. The incidence rates of liver cancer for API and NA are 2.9 and 1.2 times the incidence rate of the white population, respectively. The incidence rates of stomach cancer for API and NA are 2.2 and 1.4 times the incidence rate of white groups, respectively. The mortality rates from liver cancer for API and NA men are 2.7 and 1.6 times the mortality rate of white men, respectively. The mortality rates from stomach cancer for API and NA men are 2.0 and 1.2 times the incidence rate of white men, respectively. ${ }^{10}$

Finally, it is well reported that people from lower socioeconomic groups, irrespective of race, experience higher prevalence and mortality rates 
from cancer than people from higher socioeconomic groups In an economically homogenous group of low income people in Maryland, Mullins and colleagues ${ }^{11}$ found that the prevalence rates for lung (75/10 000), colorectal (63/ $10000)$, breast (92/10 000), and prostate $(45 / 10000)$ cancers were between 1.2 and 5.2 times the rates reported at the national level. In a national study examining socioeconomic patterns of cancer mortality in the USA between 1950 and 1998, Singh and colleagues ${ }^{12}$ found that as recently as 19971998, cancer mortality among American men was 19\% higher in communities with the low socioeconomic status (SES) than in communities with the high SES.

ISSUES OF ACCESS AND HEALTH CARE IN THE USA The causes of these disparities are only beginning to be understood. However, it is now believed that poor access to both preventive and treatment services - that is, activities that span the entire continuum of medical care-is critical to cancer control and prevention. ${ }^{413}$ For instance, patient education and counselling provides at risk people with awareness and knowledge of cancer warning signs and recommended actions to take when warning signs are present. Early detection and screening activities are important for early detection and increases cure rates for certain cancers such as cervical or colon cancers. Receiving evidence based cancer treatment can improve the quality of life and extend the lives of those stricken with the disease. ${ }^{14}$ The ability to access these services is therefore crucial to the provision of quality care.

Access to health care refers to a person's ability to obtain health services capable of bringing about the best possible health outcomes. ${ }^{13}{ }^{15}$ The ability to access health care services is the sine qua non of the health care system. Even the best health care system does a person no good if they are effectively barred from participation in that system. Several layers of health care access serve to gatekeep the system. Firstly, people must be able to obtain services in a timely fashion. Secondly, they must be able to obtain the level of services appropriate for their needs. Thirdly, they need access to continuity of care in the services they receive. We maintain that access to health care is influenced by a complicated interaction between factors involving patients, health care providers, and systems of care. ${ }^{13}{ }^{14}$ In the following discussion, we identify key factors related to access to health care and illustrate how these factors influence cancer disparities among racial/ethnic minorities and people from low income groups in the USA. The factors discussed in this review are drawn from the recent Institute of Medicine's (IOM) National Health Care Disparities Report. ${ }^{14}$

\section{FACTORS AFFECTING ACCESS TO QUALITY CANCER CARE}

Insurance status is one of the most salient factors known to influence all three levels of access to health care in the USA. ${ }^{13-15}$ The lack of a national health insurance programme means that most Americans pay for health care through a patchwork of private or public health insurance plans. Although most Americans have health insurance, about 45 million do not. Overall $70 \%$ of Americans are covered through private insurance, $26 \%$ through public (government) insurance, and $15 \%$ are uninsured. ${ }^{16}$

Private health insurance is individual or group based. Group based insurance, which is generally offered to people and their families as a benefit of employment, is by far the most common type of private health insurance held by working aged Americans (18-64 years). In 2002, nearly 10 times more working aged Americans were covered by employer sponsored insurance plans (163.7 million) compared with those covered by individual plans (16.5 million).

There are two types of public health insurance in the USA. Medicare is a federally funded public health insurance programme designed for the elderly ( $\geqslant 65$ years) and the permanently disabled. Medicare beneficiaries are supplied with hospital insurance (part A) and supplemental medical insurance (part B). In 2003, over 40 million Americans were Medicare beneficiaries. ${ }^{16}$ Medicaid is a joint federal and state funded insurance programme, run by the states, which is designed for people and families with low incomes. Each state decides how their individual Medicaid programmes will be structured, and Medicaid programmes vary significantly in terms of who is eligible for coverage, the types of services covered, and rates of payment for services. In 2003, nearly 42 million Americans were Medicaid beneficiaries. ${ }^{17}$ Between 1999 and 2003, the percentage of adults in the USA with public health insurance increased, while those with private health insurance decreased. ${ }^{16}$

Cost sharing between insurers and the insured is a central aspect of health insurance in the USA. Most of this cost sharing takes place in the form of coinsurance, copayments, and deductibles. ${ }^{17}$ Generally, there is more cost sharing between privately insured people and their insurers than between those insured through public health insurance and the federal and state governments. ${ }^{17} 18$

Within the system described above, people using the American health care system can be classified as insured,

Table 1 Leading sites of cancer deaths, female cases by race/ethnicity, USA, 2003 estimates

\begin{tabular}{|c|c|c|c|c|c|}
\hline \multicolumn{2}{|l|}{ All races } & \multicolumn{2}{|l|}{ African American } & \multicolumn{2}{|l|}{ Hispanic } \\
\hline Tumour site & Cases $(\%)$ & Tumour site & Cases (\%) & Tumour site & Cases (\%) \\
\hline All sites & $270600(100)$ & All sites & $30500(100)$ & All sites & $10100(100)$ \\
\hline Lung and bronchus & $68800(25)$ & Lung and bronchus & $6300(21)$ & Breast & $1600(16)$ \\
\hline Breast & $39800(15)$ & Breast & 5700 (19) & Lung and bronchus & 1300 (13) \\
\hline Colon and rectum & $28800(11)$ & Colon and rectum & 3700 (12) & Colon and rectum & 1100 (11) \\
\hline Pancreas & $15300(6)$ & Pancreas & $1900(6)$ & $\begin{array}{l}\text { Pancreas } \\
\text { Paris }\end{array}$ & $600(6)$ \\
\hline Ovary & $14300(5)$ & Ovary & $1100(4)$ & $\begin{array}{l}\text { Liver and intrahepatic bile } \\
\text { duct }\end{array}$ & $500(5)$ \\
\hline Non-Hodgkin lymphoma & $11200(4)$ & Multiple myeloma & $1,000(3 \%)$ & Stomach & $500(5)$ \\
\hline Leukaemia & $9800(4)$ & Uterine corpus & $1000(3)$ & Non-Hodgkin lymphoma & $500(5)$ \\
\hline Uterine corpus & $6800(3)$ & Stomach & 1000 (3) & Ovary & $500(5)$ \\
\hline $\begin{array}{l}\text { Brain and other nervous } \\
\text { system }\end{array}$ & $5800(2)$ & Uterine cervix & $800(3)$ & Uterine corpus & $300(3)$ \\
\hline Multiple myeloma & $5500(2)$ & Non-Hodgkin lymphoma & $800(3)$ & $\begin{array}{l}\text { Brain and other nervous } \\
\text { system }\end{array}$ & $300(3)$ \\
\hline All other sites & $64500(24)$ & All other sites & $7200(24)$ & All other sites & $2900(29)$ \\
\hline
\end{tabular}

Source: Surveillance, Epidemiology, and End Results Program, 1973-1999, Division of Cancer Control and Population Sciences, National Cancer Institute, 2002. As found in American Jemal et al, ${ }^{2}$ and American Cancer Society. ${ }^{79}$ 
Table 2 Leading sites of cancer deaths, male cases by race/ethnicity, USA, 2003 estimates

\begin{tabular}{|c|c|c|c|c|c|}
\hline \multicolumn{2}{|l|}{ All races } & \multicolumn{2}{|l|}{ African American } & \multicolumn{2}{|l|}{ Hispanic } \\
\hline Tumour site & Cases $(\%)$ & Tumour site & Cases $(\%)$ & Tumour site & Cases $(\%)$ \\
\hline All sites & $285900(100)$ & All sites & $32600(100)$ & All sites & $12,000(100 \%)$ \\
\hline Lung and bronchus & $88400(31)$ & Lung and bronchus & $9500(29)$ & Lung and bronchus & $2600(22)$ \\
\hline Prostate & $28900(10)$ & Prostate & $5300(16)$ & Colon and rectum & $1200(10)$ \\
\hline Colon and rectum & $28300(10)$ & Colon and rectum & 3300 (10) & $\begin{array}{l}\text { Prostate } \\
\end{array}$ & 1200 (10) \\
\hline Pancreas & $14700(5)$ & Pancreas & $1600(5)$ & $\begin{array}{l}\text { Liver and intrahepatic bile } \\
\text { duct }\end{array}$ & $900(8)$ \\
\hline Non-Hodgkin lymphoma & $12200(4)$ & Stomach & $1200(4)$ & Stomach & $800(7)$ \\
\hline Leukaemia & $12100(4)$ & Liver & 1100 (3) & Pancreas & $700(6)$ \\
\hline Oesophagus & $9900(4)$ & Oesophagus & $1000(3)$ & Non-Hodgkin lymphoma & $500(4)$ \\
\hline Liver & 9200 (3) & Multiple myeloma & $900(3)$ & Kidney & $500(4)$ \\
\hline Urinary bladder & $8600(3)$ & Non-Hodgkin lymphoma & 800 (3) & Urinary bladder & 300 (3) \\
\hline Kidney & $7400(3)$ & Oral cavity & $800(3)$ & $\begin{array}{l}\text { Brain and other nervous } \\
\text { system }\end{array}$ & $300(3)$ \\
\hline All other sites & $66200(23)$ & All other sites & $7100(22)$ & All other sites & $3000(25)$ \\
\hline
\end{tabular}

Source: Surveillance, Epidemiology, and End Results Program, 1973-1999, Division of Cancer Control and Population Sciences, National Cancer Institute, 2002. As found in American Jemal et al, ${ }^{2}$ and American Cancer Society. ${ }^{79}$

underinsured, or uninsured. The insured include people who have insurance plans that cover all needed health services who also can afford the cost sharing associated with covered benefits. The underinsured include people who have some form of health insurance but lack coverage for certain procedures or cannot afford the cost sharing associated with covered benefits, or both. Uninsured people include those who are without any form of health insurance. Americans classified as insured are viewed as having unrestricted access to the health care system. Those classified as underinsured are viewed as having limited access to health care, while those classified as uninsured are viewed as having the most restricted access to care.

Insurance related issues affect access to cancer care in a number of ways. Firstly, because participant's required contributions to medical plans have increased over the years, it is difficult for many able bodied, working aged Americans to access cancer care because they cannot afford their portion of the cost sharing associated with having health insurance. Over the past decade, more employees were required to contribute more dollars towards health insurance for themselves and their dependants. From 1992 to 2003, the percentage of private sector employees required to make contributions to their insurance plans increased from 54\% to $78 \%$ for people with single coverage, and from $74 \%$ to $90 \%$ for people with family coverage. ${ }^{18}$ During that same period, the average monthly flat rate that private sector employees were required to make for health insurance coverage increased by about $75 \%$ from $\$ 34$ to $\$ 60$ for people with single coverage, and from \$131 to \$228 for people with family coverage. ${ }^{18}$

Likewise, from 1992 to 1998, the percentage of state and local government employees who were required to make premium payments to participate in medical plans increased from $43 \%$ to $51 \%$ for those with individual coverage, and from $72 \%$ to $75 \%$ for those with family coverage. ${ }^{18}$ During this same reporting period, the average monthly dollar amount that state and local government employees were required to contribute to their medical insurance plans increased from $\$ 29$ to \$32 for people with single coverage, and from \$139 to $\$ 152$ for people with family coverage. ${ }^{18}$

As American's share for health insurance continues to increase and more Americans become uninsured or underinsured, it will become increasingly difficult to reach the objectives for cancer screening found in the document Healthy People 2010, which can ultimately lead to success in meeting National Cancer Institute's challenge of eliminating suffering and death attributable to cancer by 2015 .

Much of the excess morbidity and mortality may be attributable to a lack of access to cancer prevention and early screening technologies. The results from several studies have shown strong correlations between insurance status and participation in cancer screening activities. ${ }^{19-21}$ One of the largest and most recent studies to illustrate this association did so with an analysis of survey data from over 32000 respondents collected in the 2000 version of the National Health Interview Survey. In this national level study, Swan

Table 3 Leading sites of new cancers, female cases by race/ethnicity, USA, 2003 estimates

\begin{tabular}{|c|c|c|c|c|c|}
\hline \multicolumn{2}{|l|}{ All races } & \multicolumn{2}{|l|}{ African American } & \multicolumn{2}{|l|}{ Hispanic } \\
\hline Tumour site & Cases $(\%)$ & Tumour site & Cases $(\%)$ & Tumour site & Cases (\%) \\
\hline All sites & $658800(100)$ & All sites & $63900(100)$ & All sites & $36300(100)$ \\
\hline Breast & $211300(32)$ & Breast & 20000 (31) & Breast & 11000 (30) \\
\hline Lung and bronchus & $80100(12)$ & Lung and bronchus & $8400(13)$ & Colon and rectum & $3300(9)$ \\
\hline Colon and rectum & $74700(11)$ & Colon and rectum & $8300(13)$ & Lung and bronchus & $2200(6)$ \\
\hline Uterine corpus & $40100(6)$ & Uterine corpus & $2800(4)$ & Uterine cervix & $2100(6)$ \\
\hline Ovary & $25400(4)$ & Uterine cervix & $2100(3)$ & Uterine corpus & $1900(5)$ \\
\hline Non-Hodgkin lymphoma & $25100(4)$ & Pancreas & 2000 (3) & Thyroid & 1600 (4) \\
\hline Melanoma-skin & 24300 (3) & Ovary & $2000(3)$ & Ovary & $1500(4)$ \\
\hline Thyroid & $16300(3)$ & $\begin{array}{l}\text { Non-Hodgkin } \\
\text { lymphoma }\end{array}$ & $1700(3)$ & $\begin{array}{l}\text { Non-Hodgkin } \\
\text { lymphoma }\end{array}$ & $1300(4)$ \\
\hline Pancreas & $15800(2)$ & Kidney & $1600(3)$ & Stomach & $900(2)$ \\
\hline Urinary bladder & $15200(2)$ & Multiple myeloma & $1500(2)$ & Pancreas & $900(2)$ \\
\hline All other sites & $64500(20)$ & All other sites & $13500(21)$ & All other sites & $9600(26)$ \\
\hline
\end{tabular}

Source: Surveillance, Epidemiology, and End Results Program, 1973-1999, Division of Cancer Control and Population Sciences, National Cancer Institute, 2002. As found in American Jemal et al, ${ }^{2}$ and American Cancer Society. ${ }^{79}$ 
Table 4 Leading sites of new cancers, male cases by race/ethnicity, USA, 2003 estimates

\begin{tabular}{|c|c|c|c|c|c|}
\hline \multicolumn{2}{|l|}{ All races } & \multicolumn{2}{|l|}{ African American } & \multicolumn{2}{|l|}{ Hispanic } \\
\hline Tumour site & Cases (\%) & Tumour site & Cases (\%) & Tumour site & Cases (\%) \\
\hline All sites & $675300(100)$ & All sites & $68800(100)$ & All sites & $31100(100)$ \\
\hline Prostate & 220900 (33) & Prostate & 27000 (39) & Prostate & $8500(27)$ \\
\hline Lung and bronchus & 91800 (14) & Lung and bronchus & $10700(16)$ & Colon and rectum & 3700 (12) \\
\hline Colon and rectum & 72800 (11) & Colon and rectum & $6500(9)$ & Lung and bronchus & $2300(7)$ \\
\hline Urinary bladder & $42200(6)$ & Non-Hodgkin lymphoma & $2900(4)$ & Stomach & $1400(5)$ \\
\hline Melanoma-skin & 29900 (4) & Oral cavity & $2200(3)$ & Kidney & $1400(5)$ \\
\hline Non-Hodgkin lymphoma & 28300 (4) & Kidney & 2000 (3) & Urinary bladder & $1200(4)$ \\
\hline Kidney & $19500(3)$ & Urinary bladder & $1900(3)$ & $\begin{array}{l}\text { Liver and intrahepatic bile } \\
\text { duct }\end{array}$ & 1200 \\
\hline Oral cavity & $18200(3)$ & Pancreas & $1800(3)$ & Non-Hodgkin lymphoma & $1100(4)$ \\
\hline Leukaemiá & 17900 (3) & Stomach & $1700(3)$ & Testis & $900(3)$ \\
\hline Pancreas & $14900(2)$ & Liver & $1300(2)$ & $\begin{array}{l}\text { Brain and other nervous } \\
\text { system }\end{array}$ & $800(3)$ \\
\hline All other sites & 118900 (17) & All other sites & $10800(15)$ & All other sites & $8600(26)$ \\
\hline
\end{tabular}

Source: Surveillance, Epidemiology, and End Results Program, 1973-1999, Division of Cancer Control and Population Sciences, National Cancer Institute, 2002. As found in American Jemal et al, ${ }^{2}$ and American Cancer Society. ${ }^{79}$

and colleagues ${ }^{22}$ showed that there are strong associations between insurance status and income, and patterns of use for Pap test, mammography, prostate specific antigen (PSA) test, and home stool blood tests and colorectal endoscopy use. For the Pap test, only $62.4 \%$ of women without health insurance reported having the procedure during the previous three years compared with $79.2 \%$ of women with public insurance and $85.8 \%$ of women with private insurance. For mammography, just $38.4 \%$ of women without health insurance reported having the procedure within the previous two years compared with $61.7 \%$ of women with public insurance and $75.2 \%$ of women with private insurance. For the PSA test, only $12.6 \%$ of men without health insurance reported having the procedure within the previous year compared with 33.3\% of men with public health insurance and $44.3 \%$ of men with private health insurance. For the home blood stool test or endoscopy, just $19.8 \%$ of women and men without health insurance reported having a home blood stool test within the previous year or a colorectal endoscopy within the previous five years compared with $35.3 \%$ of women and men with public insurance and $43.8 \%$ of women and men with private insurance.

The results from this study's analyses investigating relations between SES and screening practices show that there are positive associations between SES, which was computed as an index of poverty status, and participation in all screening procedures. ${ }^{22}$ For the Pap test, just $74.2 \%$ of women with the lowest SES ( $<200 \%$ below poverty status) reported having the procedure in the previous three years compared with $90.5 \%$ of women with the highest SES ( $\geqslant 500 \%$ of poverty status). For mammography, only $55.8 \%$ of women with the lowest SES reported having the procedure compared with $82.8 \%$ of women with the highest SES. For the PSA test, just $31.7 \%$ of men with the lowest SES reported having the procedure within the past year compared with $54.3 \%$ of men with the highest SES. For the home blood stool test or colorectal endoscopy, only $33.8 \%$ of men and women with the lowest SES reported having either of the procedures within the past five years compared with $53.2 \%$ of women and men with the highest SES.

As evidenced by these studies, having health insurance helps ensure entrance to first layer of access (entry into access to the health care system), but access to the health care system does not guarantee access to the second layer of care. The type of health insurance you have can serve as a barrier to receiving a level of cancer care that is appropriate for your needs. In 2000, the Public Health Service (PHS) updated the 1996 smoking cessation guidelines developed by the Agency for Health Care and Policy Research to include some form of group or individual counselling and the use of one or more of five possible prescription or over the counter smoking cessation treatments. ${ }^{23}$ In a national study examining the extent to which states required insurance coverage for smoking cessation treatment for state employees per the revised PHS guidelines, Burns and colleagues ${ }^{24}$ discovered that $64 \%$ (29 of 45 ) of the states surveyed required only minimal coverage (at least one PHS recommended smoking cessation treatment), and just 34\% (17 of 45) provided coverage that was fully consistent with PHS recommendations. Thus, employees living in states that do not offer insurance coverage per the revised PHS guidelines for smoking cessation treatment are at an increased risk for lung cancer because their insurance status bars them from receiving a level of treatment that has been deemed appropriate for their lung cancer prevention and control needs.

Because public and private health insurance has different arrangements for the types and quantities of health services for which they will provide reimbursement, there is a wide range of variability in non-reimbursable, out of pocket expenses incurred by privately and publicly insured people undergoing cancer care. When the out of pocket expenses associated with receiving cancer care exceed a patient's ability to pay, insured people become underinsured, and a barrier to the third level of cancer care emerges-patients become unable to maintain continuity in the cancer care they are receiving.

Studies examining out of pocket expenses associated with breast cancer treatment estimate the mean range of these expenses at $\$ 360-\$ 1455$ per month. ${ }^{25-27}$ In the study with highest out of pocket estimates, ${ }^{27}$ the indirect cost associated with treatment (for example, missed wages or other income) were calculated in addition to the direct medical costs (for example, copayments) and direct non-medical costs (for example, transportation, hotel stays, meals). As might be expected, the impact of non-reimbursable, out of pocket cancer related expenses tends to be greatest among people with lower income than those with higher income. Using a sample of breast cancer patients receiving cancer care from the Comprehensive Cancer Center at Northwestern University, Arozullah and colleagues, ${ }^{27}$ found that the financial burden of breast cancer accounted for a mean $98 \%, 41 \%$, and $26 \%$ of monthly income among women with annual household income levels of $\leqslant \$ 30000$, \$30 00160000 , and $>\$ 60000$, respectively. The most commonly reported out of pocket expenditures reported among this sample were for drugs $(80 \%)$, transportation $(78 \%)$, physician visits $(66 \%)$, and restaurant meals $(51 \%)$. 


\section{Key references}

- National Institutes of Health. NIH strategic research plan to reduce and ultimately eliminate health disparities. US Department of Health and Human Services. http://www.nih.gov/about/hd/strategicplan.pdf (accessed 18 Oct 2004).

- Agency for Healthcare Research and Quality, Department of Health and Human Services. National healthcare disparities report. Rockville, MD: Agency for Healthcare Research and Quality, 2003.

- Bradley CJ, Given CW, Roberts C. Disparities in cancer diagnosis and survival. Cancer 2001;91:178-88.

- Swan J, Breen N, Coates RJ, Rimer BK, et al. Progress in cancer screening practices in the United States: results from the 2000 National Health Interview Survey. Cancer 2003;97:1528-40.

- Anderson LM, Scrimshaw SC, Fullilove MT, et al. Culturally competent healthcare systems. A systematic review. Am J Prev Med 2003;24(suppl 1):68-79.

\section{CULTURALLY BASED BARRIERS TO CARE}

Culture refers to integrated patterns of beliefs, values, and lifestyles that have developed among racial/ethnic and social groups to ensure the survival and wellbeing of its members. ${ }^{28}{ }^{29}$ Culture has an important influence on many aspects of health. The culture of any given group helps determines what it means to be healthy, it influences acceptable methods for attaining, maintaining, or regaining health, and it influences how health and illness are described. ${ }^{28}$ There is a growing body of research that suggests that many Americans experience barriers within the context of medical encounters that negatively affect their ability to navigate within the health care system that can be explained by cultural factors. Communication, stereotyping, bias, and discrimination are among the most well researched culturally based barriers that influence access to cancer care for Americans from ethic/ racial minority groups and from low income groups.

Communication is an important aspect of culture and an essential component of any physician-patient encounter. It is through communication that patients present symptoms, physicians explain treatment options, physicians describe treatment regimens, patients express approval or disapproval with physicians' recommendations, and patients show understanding of medical instructions. Because the demographics of Americans seeking medical care have changed more dramatically than the demographics of Americans delivering care, people from racial/ethnic minorities groups are more likely to experience culturally discordant physicianpatient encounters than white people. For instance, African Americans and Hispanics comprise $12.9 \%$ and $12.5 \%$ of the US population, respectively. ${ }^{30}$ Yet, African Americans and Hispanics comprise only $2.4 \%$ and $3.3 \%$ of total proportion of physicians in the USA, respectively. ${ }^{31}$

The literature on physician-patient relationships is replete with studies showing that physician-patient communication problems because of cultural differences is commonplace. ${ }^{32-38}$ Many communication problems occur because physicians fail to detect patient's direct or indirect inquiries for information and/or physicians are unable to communicate back to patients, in language clear to patients, their medical diagnosis, their treatment options, or how treatment alternatives might relate to patients' system of values. ${ }^{34}$

Communication issues, like those mentioned above, create a sequence of events that can affect access to care in the following manner. Physicians and patients who experience communication difficulties during medical encounters find it harder to establish a good rapport. In medical encounters where a good rapport is not established, it will be more difficult for physicians and patients to establish trust. Patients who do not trust physicians are more likely to be less communicative and more likely to rate the quality of their medical encounter as unsatisfactory. Patients who rate the quality of their medical encounters as unsatisfactory are less likely to be compliant with medical recommendations, more likely to delay seeking care in the future, or more likely to seek care from alternative sources. ${ }^{35}$

Examples of communication serving as a barrier to cancer control and prevention are found throughout the literature. In a study undertaken to understand the breast cancer experiences of Asian American women, Ashing et $a l^{39}$ found that newly immigrated patients who lacked fluency in speaking English were reluctant to request additional breast cancer information, beyond that which was given, when they were paired with physicians who did not speak the patient's primary language. In another study looking at physician initiated discussions of cancer screening tests with patients, Dunn and colleagues ${ }^{40}$ reported that physicians mentioned language barriers between patients and themselves among the top three reasons for them not discussing PSA testing and mammography with patients. Care that is not culturally competent can result in missed and delayed diagnoses of cancer and inadequate treatment for patients with cancer. Moreover, cancer patients' quality of life can be compromised during the course of treatment and into survivorship.

\section{CONCLUSION}

In this review, we described three layers of health care access that are essential to comprehensive cancer care. The factors that facilitate or inhibit access at each level are intertwined. Different obstacles must be overcome before the health care systems of UK or the USA can offer comprehensive cancer control to all of their citizens. The UK's national health insurance plan guarantees access to secondary preventive services (for example, cancer screening) and ensures continuity in the levels of cancer care they receive through the NHS. However, the 2000 NHS Caner Plan (http://www.doh. gov.uk/cancer) suggests that NHS consumers do not always receive the level of cancer care appropriate for their cancer control needs because of a shortage of cancer specialists, their unequal geographical distribution, and the need to update equipment in many areas.

By contrast, cancer care in the USA is among the most technically outstanding in the world. However, the US healthcare system is at a crossroads as it struggles to provide care to all its citizens. Significant numbers of Americans are either underinsured or uninsured. This limits access to the complete range of cancer care services including and especially those directed at primary prevention. This unequal access amounts to a form of rationing. Finally, the US population is the most culturally diverse in the developed world. The heterogeneity of the population increases the challenges to the health care system to provide excellent cancer care to all who need it.

\section{MULTIPLE CHOICE QUESTIONS (ANSWERS AT THE END OF THE REFERENCES)}

1. What ethnic group in the USA has the highest cancer mortality rate among women?

(A) African Americans

(B) White Americans

(C) Hispanics 
(D) Asians

2. What ethnic group in the USA has the highest cancer mortality rate among men?

(A) African Americans

(B) White Americans

(C) Hispanics

(D) Asians

3. How many Americans have no health insurance?

(A) 20 million

(B) 2 million

(C) 45 million

(D) 100 million

4. Good communication with patients is important to the provision of cancer care because:

(A) It can lead to early diagnosis

(B) It can increase adherence to treatment

(C) It can increase patients' trust and satisfaction

(D) All of the above

5. Americans who have no health insurance or who are underinsured are more likely, less likely, or not as likely to receive cancer preventive services?
(A) Just as likely
(B) Not as likely
(C) More likely

\section{Authors' affiliations}

L A Siminoff, Case Western Reserve University, Department of Bioethics and the Case Comprehensive Cancer Center, Cleveland, USA

L Ross, Case Western Reserve University, Case Comprehensive Cancer Center

Funding: none.

Conflicts of interest: none.

\section{REFERENCES}

1 American Cancer Society. Cancer facts and figures 2003, Publication number 5008.03. Atlanta, GA: American Cancer Society, 2003.

2 Jemal A, Murray T, Samuels A, et al. Cancer statistics, 2003. CA Cancer J Clin 2003;53:5-26.

3 National Institute of Health. NIH strategic research plan to reduce and ultimately eliminate health disparities. US Department of Health and Human Services. http://www.nih.gov/about/hd/strategicplan.pdf (accessed 18 Oct 2004)

4 Centers for Disease Control, Prevention (CDC). Guidance for comprehensive cancer control planning volume 1: guidelines. Atlanta, GA: US Department of Health and Human Services, 2002.

5 Ghafoor A, Jemal A, Cokkinides V, et al. Cancer statistics for African Americans. CA Cancer J Clin 2002;52:326-41.

6 Ries L, Eisner MP, Kosary CL, et al. SEER cancer statistics review, 1975-2000. Publication number 00-2789. Bethesda, MD: National Cancer Institute, 2003

7 American Cancer Society. Cancer facts and figures for African Americans 2003-2004. Atlanta, GA: American Cancer Society, 2003.

8 United States Census Bureau. US interim projections by age, sex, race, and Hispanic origin. http://www.census.gov/ipc/www/usinterimproj/ natprojtab0 la.pdf (accessed 10 Nov 2004).

9 American Cancer Society. Cancer facts and figures for Hispanics/Latinos 2003-2005. Atlanta, GA: American Cancer Society, 2003.
10 National Cancer Institute. Cancer health disparities: fact sheet. http:// www.cancer.gov/newscenter/healthdisparities (accessed 7 Nov 2004).

11 Mullins CD, Cooke JL Jr, Wang J, et al. Disparities in prevalence rates for lung, colorectal, breast, and prostate cancers in Medicaid. I Natl Med Assoc 2004;96:809-16.

12 Singh GK, Miller BA, Hankey BF. Changing area socioeconomic patterns in US cancer mortality, 1950-1998: part II-Lung and colorectal cancers. J Natl Cancer Inst 2002;94:916-25.

13 US Department of Health and Human Services. Healthy people 2010: understanding and improving health. 2nd ed. Washington, DC: US Government Printing Office, 2000.

14 Agency for Healthcare Research and Quality, US Department of Health and Human Services. National healthcare disparities report. Rockville, MD: Agency for Healthcare Research and Quality, 2003.

15 Cockerham WC. Medical sociology. 7th ed. Upper Saddle River, NJ: Prentice Hall, 1998.

16 United States Census Bureau. Health insurance coverage in the United States: 2002: US Department of Commerce, 2003.

17 Kaiser Family Foundation/eHealth Insurance. Update on individual health insurance. Washington, DC: Kaiser Family Foundation, 2004.

18 Baker CA. Cost sharing in medical insurance plans. US Department of Labor, Bureau of Labor Statistics. http://www.bls.gov/opub/cwc/ cm20040326ar01pl.htm (accessed 5 Oct 2004).

19 Bradley CJ, Given CW, Roberts C. Disparities in cancer diagnosis and survival. Cancer 2001;91:178-88.

20 Roetzheim RG, Pal N, Tennant C, et al. Effects of health insurance and race on early detection of cancer. J Natl Cancer Inst 1999;91:1409-15.

21 Ayanian JZ, Kohler BA, Abe T, et al. The relation between health insurance coverage and clinical outcomes among women with breast cancer. N Engl J Med 1993;329:326-31

22 Swan J, Breen N, Coates RJ, et al. Progress in cancer screening practices in the United States: results from the 2000 National Health Interview Survey. Cancer 2003;97:1528-40.

23 Task Force on Community Preventive Services. Recommendations regarding interventions to reduce tobacco use and exposure to environmental tobacco. Am J Prev Med 2001;20(suppl 2):10-15.

24 Burns ME, Bosworth TW, Fiore MC. Insurance coverage of smoking cessation treatment for state employees. Am J Public Health 2004;94:1338-40.

25 Given BA, Given CW, Stommel M. Family and out-of-pocket costs for women with breast cancer. Cancer Pract 1994;2:187-93.

26 Moore KA. Breast cancer patients' out-of-pocket expenses. Cancer Nurs 1999;22:389-96.

27 Arozullah AM, Calhoun EA, Wolf M, et al. The financial burden of cancer: estimates from a study of insured women with breast cancer. I Support Oncol 2004;2:271-8.

28 Kagawa-Singer M, Kassim-Lakha S. A strategy to reduce cross-cultural miscommunication and increase the likelihood of improving health outcomes. Acad Med 2003;78:577-87.

29 Anderson LM, Scrimshaw SC, Fullilove MT, et al. Culturally competent healthcare systems. A systematic review. Am J Prev Med 2003;24(suppl 3):68-79.

30 United States Census Bureau. Profile of general demographic characteristics: 2000, census 2000 summary file 1. http://factfinder.census.gov/servlet/ (accessed 13 Nov 2004).

31 American Medical Association. Physician characteristics and distribution in the US. Chicago, IL: American Medical Association, 2004.

32 Cooper-Patrick L, Gallo JJ, Gonzales JJ, et al. Race, gender, and partnership in the patient-physician relationship. JAMA 1999;282:583-9.

33 Van Ryn M, Burke J. The effect of patient race and socio-economic status on physicians' perceptions of patients. Soc Sci Med 2000;50:813-28.

34 Doescher MP, Saver BG, Franks P, et al. Racial and ethnic disparities in perceptions of physician style and trust. Arch Fam Med 2000;9:1 156-63.

35 Ferguson WJ, Candib LM. Culture, language, and the doctor-patient relationship. Fam Med 2002;34:353-61.

36 Butow PN, Brown RF, Cogar S, et al. Oncologists' reactions to cancer patients' verbal cues. Psycho-Oncology 2002; 1 1:47-58

37 Cooper LA, Roter DL, Johnson RL, et al. Patient-centered communication, ratings of care, and concordance of patient and physician race. Ann Intern Med 2003; 139:907-15.

38 Johnson RL, Saha S, Arbelaez JJ, et al. Racial and ethnic differences in patient perceptions of bias and cultural competence in health care. J Gen Intern Med 2004;19:101-10

39 Ashing KT, Padilla G, Tejero J, et al. Understanding the breast cancer experience of Asian American women. Psycho-Oncology 2003;12:38-58.

40 Dunn AS, Shridharani KV, Lou W, et al. Physician-patient discussions of controversial cancer screening tests. Am J Prev Med 2001;20:130-4.

\section{ANSWERS}

1. (A); 2. (A); 3. (C); 4. (D); 5. (B). 